\title{
Test of $\phi$ renormalization in nuclei through $\phi$ photoproduction
}

\author{
E. Oset ${ }^{1}$, M.J. Vicente Vacas ${ }^{1}$, H.Toki ${ }^{2}$, A. Ramos ${ }^{3}$ \\ 1 Departamento de Física Teórica and IFIC, \\ Centro Mixto Universidad de Valencia-CSIC, \\ Ap. Correos 22085, E-46071 Valencia, Spain \\ ${ }^{2}$ Research Center for Nuclear Physics, Osaka University, Ibaraki, \\ Osaka 567-0047, Japan \\ ${ }^{3}$ Departament d'Estructura i Constituents de la Matèria, \\ Universitat de Barcelona, \\ Diagonal 647, E-08028 Barcelona, Spain
}

\begin{abstract}
We propose an experimental procedure to find out the medium modifications of the $\phi$ meson. The reaction is inclusive $\phi$ photoproduction in nuclei, looking for $K^{+} K^{-}$pairs from the $\phi$ decay with total momentum smaller than 100-150 $\mathrm{MeV} / \mathrm{c}$, which are made possible at energies of present laboratories from center of mass $\phi$ backward production and the help of Fermi motion. We have conducted a many body calculation of the mass distribution of the $\phi$ adapted to the experimental set up of a recent JLAB experiment where the backwards $\phi$ photoproduction has been measured. Using recent results for the in medium properties of the $\phi$, we find that the width of the invariant mass distribution for photoproduction on medium to heavy nuclei is larger than the free $\phi$ width by a factor of two or more.
\end{abstract}

PACS: 13.25.-k, 13.75.Jz, 14.40.Cs

Keywords: $\phi$ decay in nuclei, Chiral Lagrangians, $\bar{K}$-nucleus interaction. 
The medium modification of vector mesons in nuclei is the subject of intense study, particularly for the $\rho$ meson, both theoretical [1, 2, 3, 4, 5] and experimental [6, 7]. The search for the medium modifications proceeds experimentally mostly by means of back to back dilepton production in heavy ion reactions, which ensures the $\rho$ production at rest and guarantees its decay inside the nucleus. Yet, experiments are difficult to interpret since they require to include a cocktail of background reactions out of which the $\rho$ contribution is only a fraction of the total, and the present measurements are explained both in terms of a drop of the $\rho$ mass [8], as well as with just an appreciable increase in the decay width [3].

The $\phi$ meson has also been the subject of some works [4, 9, 10, 11, 12, 13] and the results of these papers point at a sizeable renormalization of the $\phi$ width and a small mass shift. Testing the $\phi$ properties in a nuclear medium is an interesting problem by itself, as well as for what it bears in common with other vector mesons, which share some of the renormalization mechanisms of the $\phi$. The $\phi$ renormalization in matter has also received attention in heavy ion collisions [14, 15, 16] since $\phi$ production has been advocated as one of the signatures for a possible transition to the quark gluon plasma. Another reason is the connection with the changes of kaons inside matter, a subject of intense debate due to strong deviations from the low density theorem already at very low densities, as evidenced by the needed attraction to reproduce kaonic atom data [17, 18, 19, 20], as well as due to the possibility of having kaon condensation in neutron stars at sufficiently large densities 21.

We propose here a simple and clean method for measuring the modifications of the $\phi$ meson in nuclei. The reaction is inclusive $\phi$ photoproduction in nuclei looking only into the slow $\phi$ mesons produced. The $\phi$ photoproduction on proton targets is a subject of research in laboratories like Jefferson Lab [22], or Spring8/Osaka 23], and extensive theoretical studies have been already performed [24]. Actually the experiment planned at Spring8/Osaka is done with $\mathrm{CH} 2$ targets, and hence data on protons as well as in nuclei will be simultaneously accumulated.

The reaction proposed is $\gamma A \rightarrow \phi X$ where the elementary process is the $\gamma N \rightarrow \phi N$ reaction. If we take the $\phi$ produced backwards in the center of mass $(\mathrm{CM})$ of the elementary reaction, the $\phi$ momentum in the nucleon at rest frame (lab frame) is $235 \mathrm{MeV} / \mathrm{c}, 168 \mathrm{MeV} / \mathrm{c}$ for photon energies of $3.6 \mathrm{GeV}$ and $6 \mathrm{GeV}$, respectively, and smaller at higher photon energies. Yet, it is possible to have even smaller $\phi$ momenta in the nuclear reaction due to Fermi motion if the production takes place on nucleons carrying a momentum opposite to the one of the photon. In this case, by putting a restriction on the momenta of the outgoing $\phi$, we can guarantee that most of the $\phi$ produced would decay inside the nucleus, thus showing the medium properties of the meson. We shall see that by restricting the momentum to 
be smaller than 100-150 $\mathrm{MeV} / \mathrm{c}$ there is still a sizeable proportion of events to make the method of testing $\phi$ properties in a nuclear medium efficient from the experimental point of view. The dependence of the cross section on the invariant mass of the $\phi$ can be deduced in this case from its decay into $K^{+} K^{-}$. The distortion of the low energy kaons, of about $20 \mathrm{MeV}$ energy, can be easily handled because the quasielastic collisions are very much hindered by Pauli blocking. On the other hand, the events in which the $K^{-}$is absorbed are simply lost. This results in a decrease of the cross section without affecting much the shape of the invariant mass distribution of the $\phi$ and it will be taken into consideration in the calculations.

In order to evaluate theoretically the inclusive nuclear photoproduction cross section we apply many body techniques, successfully used in inclusive neutrino [25, 26] and photon [27] nucleus reactions. We start by evaluating the photon selfenergy in nuclear matter which accounts for $\phi-p h$ production in intermediate states, see fig.1 or analogously the $\gamma N \rightarrow \phi N$ reaction inside the nucleus. In the evaluation of the photon selfenergy we will explicitly take into account the modification of the $\phi$ properties in the medium. For this purpose we shall use the results for the mass (no change or negligible at the low density involved in this reaction) and the width from refs. 112, 13. The width contains $\phi$ decay into $K, \bar{K}$ (modified in the medium) as well as $\phi N \rightarrow K Y$ channels $(Y=\Lambda, \Sigma)$. We shall call $T$ the matrix element for the $\gamma N \rightarrow \phi N$ transition. Since we are only interested in the slow $\phi$ coming from $\phi$ produced backwards in the CM, the dynamics of the process would enter through the elementary transition matrix element backwards which we call $C$. We shall also assume it to be the same for protons and neutrons for simplicity, but even if it has different weights it would only show up in the total strength of the cross section but not in the shape of the $\phi$ invariant mass distribution, which is the one that will provide the medium properties of the $\phi$ meson.

The photon selfenergy associated to the diagram of fig. 1 is given by

$$
-i \Pi(k, \rho)=\int \frac{d^{4} q}{(2 \pi)^{4}} i U(k-q, \rho) \frac{i(-i T)(-i T)}{q_{0}^{2}-\vec{q}^{2}-m_{\phi}^{2}+i m_{\phi} \Gamma_{\phi}(\rho)}
$$

where $U(p, \rho)$ is the relativistic Lindhard function for $p h$ excitation, for which we use the analytical expression given in 25] (we multiply here the expression by a factor two, in order to account for protons and neutrons, and take the same value for the Fermi momentum of protons and neutrons, $\left(k_{F}=\right.$ $\left.\left(3 \pi^{2} \rho / 2\right)^{1 / 3}\right)$.

Making use of Cutkosky rules to evaluate the imaginary part of $\Pi$

$$
\begin{aligned}
\Pi & \rightarrow 2 i \operatorname{Im} \Pi \\
U(k-q) & \rightarrow 2 i \theta\left(k^{0}-q^{0}\right) \operatorname{Im} U(k-q) \\
D(q) & \rightarrow 2 i \theta\left(q^{0}\right) \operatorname{Im} D(q),
\end{aligned}
$$




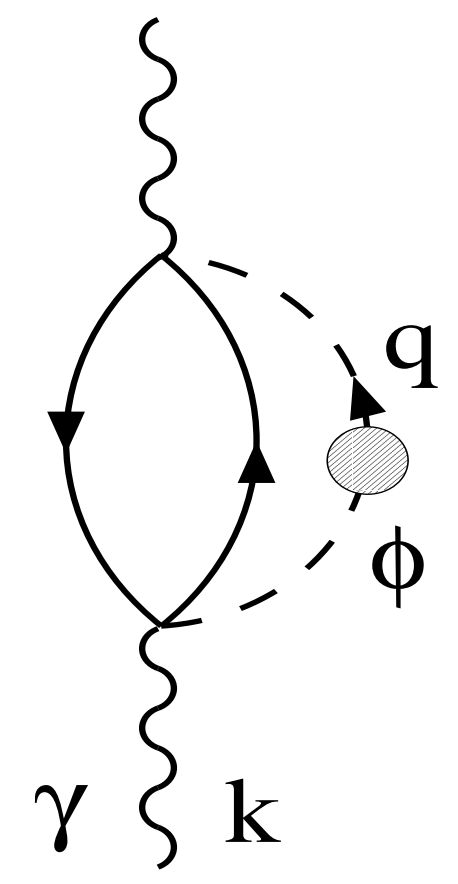

Figure 1: Photon selfenergy diagram accounting for a $\phi$ and a particle-hole in the intermediate states.

where $D(q)$ is the $\phi$ propagator, we obtain

$\operatorname{Im} \Pi(k, \rho)=\int \frac{d^{4} q}{(2 \pi)^{4}} \theta\left(k^{0}-q^{0}\right) \theta\left(q^{0}\right) \operatorname{Im} U(k-q, \rho) \frac{2|T|^{2} m_{\phi} \Gamma_{\phi}(\rho)}{\left|q_{0}^{2}-\vec{q}^{2}-m_{\phi}^{2}+i m_{\phi} \Gamma_{\phi}(\rho)\right|^{2}}$

Since $-I m \Pi / k$ gives the reaction probability per unit length for the reaction $\gamma N \rightarrow \phi N$ to occur inside the nucleus, the nuclear cross section is then given by (see also [27]):

$$
\sigma_{A}=-\int d^{3} r \frac{\operatorname{Im} \Pi(k, \rho(\vec{r}))}{k}
$$

One can see that in the limit of small densities, where the Lindhard function takes the form

$$
\operatorname{ImU}(k-q, \rho) \simeq-\frac{M}{E(\vec{k}-\vec{q})} \rho \delta\left(k^{0}-q^{0}+M-E(\vec{k}-\vec{q})\right),
$$

with $M, E(\vec{p})$ the mass and energy of the nucleon, respectively, eq. (4) leads to the intuitive result $\sigma_{A}=A \sigma$, where $\sigma$ is the elementary $\gamma N \rightarrow \phi N$ cross section . 
The differential cross section for $\phi$ photoproduction with the $\phi$ going forward in the nuclear lab frame, but with the small momenta which correspond to the backwards CM photoproduction, is given from eqs. (3),(4) by

$$
\begin{aligned}
\left.\frac{d \sigma}{d \Omega}\right|_{A} & =-\frac{1}{k} \int d^{3} r \frac{1}{(2 \pi)^{3}} \int \frac{d q^{0}}{2 \pi} \int q^{2} d q \theta\left(k^{0}-q^{0}\right) \theta\left(q^{0}\right) \\
& \times \operatorname{ImU}(k-q, \rho) \frac{2|C|^{2} m_{\phi} \Gamma_{\phi}(\rho)}{\left|q_{0}^{2}-\vec{q}^{2}-m_{\phi}^{2}+i m_{\phi} \Gamma_{\phi}(\rho)\right|^{2}}
\end{aligned}
$$

Even if we look at $\phi$ produced with small momentum, the $\phi$ will travel a certain distance inside the nucleus. The $\phi-p h$ production occurs at the point $\vec{r}$ in the nucleus and hence the argument in the Lindhard function is $\rho(r)$. However, the $\phi$ is produced in the point $\vec{r}$ but decays at a point $\vec{r}+\overrightarrow{\Delta l}$ where $\overrightarrow{\Delta l}$ is the average distance traveled by the $\phi$ in its life time in the nucleus. Thus, for the width of the $\phi$ (and eventually the density dependent mass) appearing in the $\phi$ propagator, we take the density at the average point between the production and the decay, this is at $\vec{r}^{\prime}=\vec{r}+\Delta \vec{l} / 2$, where $\overrightarrow{\Delta l}=\vec{q} / \omega(q) \Gamma(\rho)$, with $\Gamma(\rho)$ the $\phi$ width at the production point and $\vec{q}$ taken forward in the nuclear lab frame. We can make some iteration in our procedure in order to take this width also at the intermediate point and our final results are evaluated in that way.

Taking now $\vec{b}$ as the impact parameter and replacing the integration variable $d q$ by $d s\left(s=\left(q^{0}\right)^{2}-\vec{q}^{2}=M_{I}^{2}\right)$, we obtain the final formula, which accounts already for the cut in the $\phi$ momentum,

$$
\begin{aligned}
\frac{d \sigma}{d \Omega d M_{I}} & =-\frac{2}{k(2 \pi)^{3}} \int b d b \int d z \int d q^{0} \bar{q} M_{I} \theta\left(k^{0}-q^{0}\right) \theta\left(q^{0}\right) \\
& \times\left.\operatorname{ImU}(k-q, \rho(\vec{r}))\right|_{q=\bar{q}} \frac{\bar{q} M_{I}|C|^{2} m_{\phi} \Gamma_{\phi}\left(\rho\left(\vec{r}^{\prime}\right)\right) C_{F} C_{A}}{\left|q_{0}^{2}-\vec{q}^{2}-m_{\phi}^{2}+i m_{\phi} \Gamma_{\phi}\left(\rho\left(\vec{r}^{\prime}\right)\right)\right|^{2}}
\end{aligned}
$$

with

$$
\begin{aligned}
\bar{q} & =\left(q_{0}^{2}-s\right)^{1 / 2} \\
r & =\left(b^{2}+z^{2}\right)^{1 / 2} \\
r^{\prime} & =\left(b^{2}+(z+\Delta l / 2)^{2}\right)^{1 / 2} \\
\Delta l & =\frac{\bar{q}}{\omega(\bar{q})} \frac{1}{\Gamma_{\phi}(\rho(r))}
\end{aligned}
$$

with the acceptance factor, $C_{F}=0$ for $q>q_{c u t}$ and 1 otherwise. The factor $C_{A}$ is the $K^{-}$absorption factor. It is evaluated for each point $\vec{r}$ by assuming the $\phi$ to go forward. Then a random direction is taken for the $K^{-}$in the $\phi$ rest frame and the $K^{-}$momentum is boosted to the $\phi$ moving 
frame. The surviving probability of this kaon is easily evaluated knowing that the probability of absorption per unit length is $-\operatorname{Im} \Pi_{K} / p_{K}$, where $\Pi_{K}$ is the $K^{-}$selfenergy which we take from [28]. Finally an average for all the original random momenta is taken. Another source of nuclear corrections is the Coulomb correction to the $K^{+} K^{-}$which will modify the invariant mass of the reconstructed $\phi$. An estimate can be done taking a couple of kaons going back to back from the center of the nucleus, assuming about 5 $\mathrm{MeV}$ increase of energy to the $K^{+}$and an equal decrease for the $K^{-}$with the corresponding changes in the momenta. The invariant mass change with respect to the free one is less than $1 \mathrm{MeV}$. This magnitude is small compared to the strong interaction changes that we are discussing and hence we neglect it in the evaluations.

The absorption of the $K^{-}$is rather strong and only those $K^{-}$produced at the nuclear surface have a chance to survive. In our calculations the average nuclear density for these observable events is around $\rho=\rho_{0} / 4$. According to recent selfconsistent calculations of the $K^{-}$selfenergy using the chiral Lagrangians [28, 29, 30] the $K^{-}$potential at this density is of the order of $-10 \mathrm{MeV}$. These results have been found consistent with $K^{-}$atomic data [18, 19, 20]. Hence, the distortion of the $K^{-}$on their way out due to this potential is expected to be small.

We perform now calculations for a photon energy of $3.6 \mathrm{GeV}$, the energy of the experiment performed by the CLAS collaboration at Jefferson Lab [22]. The value of the constant $C$ can be obtained from the data of this experiment. Taking $d \sigma / d t=1 \mathrm{nb} / \mathrm{GeV}^{2}$ at backward angles from 22 we obtain $|C|^{2}=650 \mathrm{nb}$. One of the striking features of this experiment is the relatively large backward cross section, which is two orders of magnitude bigger than what one would expect from the extrapolation of the previous data at small angles [31, or theoretical expectations based on Pomeron exchange. On the other hand, theoretical approaches based on two gluon exchange do predict such enhancement of the backward cross section [32]. For us what matters is that this cross section is large and measurable and, provided we find nuclear cross sections of the same order of magnitude, the feasibility of the experiment is guaranteed.

Our results for the photoproduction of the $\phi$ on ${ }^{40} \mathrm{Ca}$ are shown in fig.2 at a photon energy $E_{\gamma}=3.6 \mathrm{GeV}$ and two $\phi$ momentum cuts of $100 \mathrm{MeV} / \mathrm{c}$ and $150 \mathrm{MeV} / \mathrm{c}$. The calculations have been done using the density dependent $\phi$ width obtained in [13] and a mass of the $\phi$ independent of the density. The calculations of [4, 12] indicate that the variation of the mass is of the order of one percent. The width of the $\phi$ obtained in [13] at $\rho=\rho_{0}$ is of the order of $22 \mathrm{MeV}$, and the density dependence is not exactly linear. We shall also show some cross sections (in fig. 3) using the results of [4, 12] where the $\phi$ width is about $45 \mathrm{MeV}$ at $\rho=\rho_{0}$. For comparison we also show in fig. 2 the results obtained with the free width of the $\phi$. We can see that, for a same 


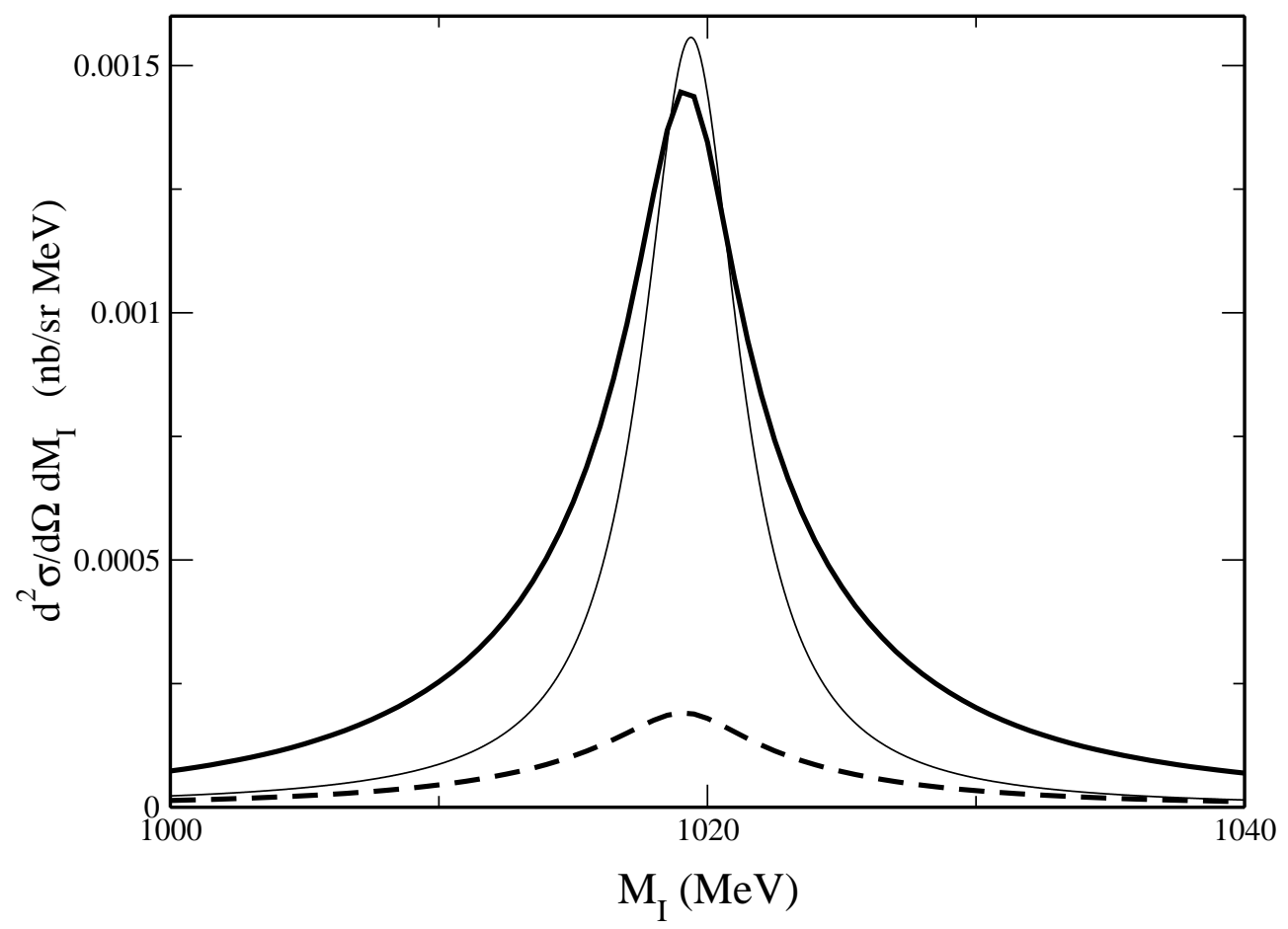

Figure 2: $\phi$ photoproduction cross section in ${ }^{40} \mathrm{Ca}$. The dashed line has a cut in the $\phi$ momentum of $100 \mathrm{MeV} / \mathrm{c}$. The thick solid line is for a cut of $150 \mathrm{MeV} / \mathrm{c}$. The thin solid line is one third of the cross section calculated using the free $\phi$ width and a cut of $150 \mathrm{MeV} / \mathrm{c}$.

momentum cut, the use of the width of the $\phi$ in the medium reduces the cross section in about a factor three and leads to a wider mass distribution.

The results of fig. 2 show an invariant mass distribution with a $\phi$ width of about $10 \mathrm{MeV}$ for the momentum cut of $100 \mathrm{MeV} / \mathrm{c}$. Increasing the momentum cut at $150 \mathrm{MeV} / \mathrm{c}$ reduces the width to about $8 \mathrm{MeV}$, nearly twice the free width, but it has the advantage to increase the cross section by about a factor five, which is desirable from the experimental point of view. This also means that moderate increases in the cut can be done, at the expense of losing some width but increasing further the strength of the cross section.

It is worth mentioning that, should we have not included the cut in the momentum of the $\phi$, the amount of events where the $\phi$ decays outside the nucleus, even concentrating on those coming from backward $\phi$ production in the CM frame, is quite large and the mass distribution has a width very close to the free one and is rather insensitive to the medium corrections. Thus, the cut in the $\phi$ momentum is an essential ingredient for the observation of the $\phi$ properties in the medium. A smaller momentum cut further stresses the 


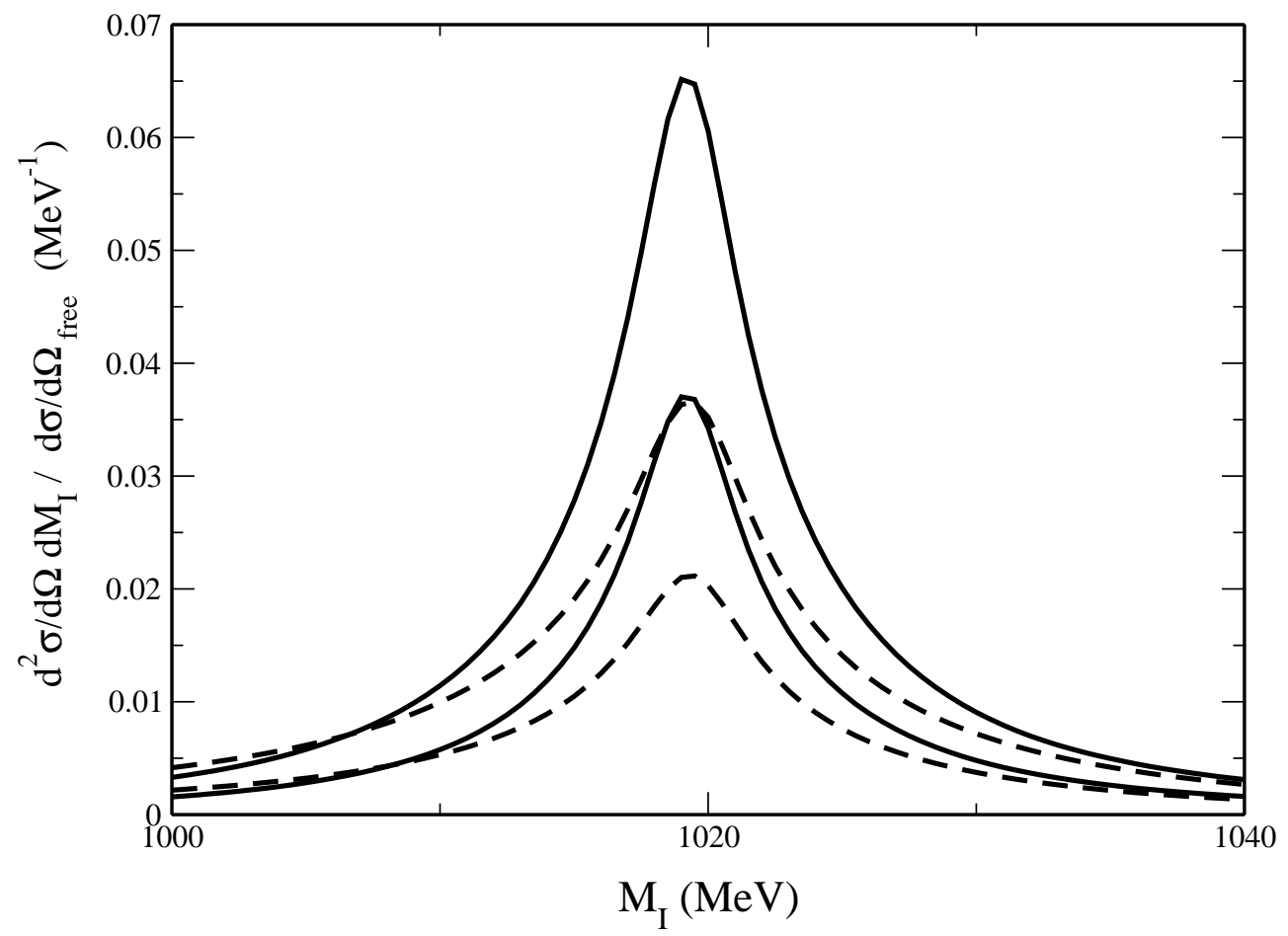

Figure 3: $\phi$ photoproduction cross section at $E_{\gamma}=3.6 \mathrm{GeV}$ normalized to the elementary one at backward angles. Solid lines: ${ }^{40} \mathrm{Ca}$ (upper) and ${ }^{16} \mathrm{O}$ (lower). Dashed lines: Results with the medium dependent width of [4, 12]

medium corrections at the cost of having fewer events, but smaller cuts than $100 \mathrm{MeV} / \mathrm{c}$ are still possible with enough statistics. With higher energies of the photon beam one has more events with low $\phi$ momentum but the backward photoproduction cross section might also decrease. Knowledge of these cross sections in a range of energies in the $\mathrm{GeV}$ region would help in finding an optimum photon energy for the present reaction.

In order to have a feeling about the feasibility of the experiment we present the results in a different way, dividing the nuclear cross section by the elementary $d \sigma / d \Omega$ cross section in the lab frame at backward CM angles, which we induce easily from the experimental value of $d \sigma / d t$ of ref. [22]. The results corresponding to fig. 2 (with the momentum cut of $150 \mathrm{MeV} / \mathrm{c}$ ) are shown in fig. 3 in units of $\mathrm{MeV}^{-1}$ for two nuclei. This is a convenient representation, since the area of the curve gives us the fraction of the elementary backward cross section that we obtain in the nuclear case with the proposed set up. We observe that we obtain a fraction of about 45 percent of the free cross section in the case of ${ }^{16} \mathrm{O}$ and about 85 percent for the case of ${ }^{40} \mathrm{Ca}$. In the same figure we also show the results obtained using the $\phi$ width in the medium of [4, [12]. We observe in this case that the width of the $\phi$ 
distribution is further increased and the strength of the cross section at the peak decreased but is still sizeable. The results shown in fig. 3 thus indicate that the experimental observation of sizeable changes in the nuclear width of the $\phi$ should require about the same time as the measurements done in [22].

In summary, the reaction proposed of inclusive $\phi$ photoproduction in nuclei, including a cut of about 100-150 MeV/c for the $\phi$ momentum, proves to be sensitive to the $\phi$ properties inside nuclear matter. The large enhancements in the $\phi$ width predicted by theoretical models, and the relatively large cross sections calculated, call for the implementation of the experiment. Furthermore, the data obtained could serve as a test of the approaches used in the renormalization of other vector mesons, like the $\rho$, and also as a further consistency test of theories about the modification of kaon properties in nuclei where there is still much debate [17, 28, 30, 33, 34, 35].

\section{Acknowledgments}

We would like to acknowledge useful discussions with V. Burkert and D. Tedeschi. This work is partly supported by DGICYT contracts no. PB 960753 and PB98-1247 and by the EEC-TMR Program Contract no. ERBFMRXCT98-0169.

\section{References}

[1] M. Herrmann, B.L. Friman and W. Norenberg, Nucl. Phys. A560 (1993) 411

[2] G. Chanfray and P. Schuck, Nucl. Phys. A555 (1993) 329

[3] R. Rapp, G. Chanfray and J. Wambach, Nucl. Phys. A617 (1997) 472

[4] F. Klingl, N. Kaiser and W. Weise, Nucl. Phys. A624 (1997) 527

[5] W. Peters, M. Post, H. Lenske, S. Leupold and U. Mosel, Nucl. Phys. A632 (1998) 109

[6] J. Friese, Prog. Part. Nucl. Phys. 42 (1999) 135

[7] G. Agakishiev et al., Phys. Lett. B422 (1998) 405 
[8] G.Q. Li, C.M. Ko, G.E. Brown and H. Sorge, Nucl. Phys. A611 (1996) 539

[9] H. Kuwabara and T. Hatsuda, Prog. Theor. Phys. 94 (1995) 1163.

[10] K. Haglin, Nucl. Phys. A584 (1995) 719

[11] W. Smith and K. L. Haglin, Phys. Rev. C 57 (1998) 1449

[12] F. Klingl, T. Waas and W. Weise, Phys. Lett. B431 (1998) 254

[13] E. Oset and A. Ramos, Nucl. Phys. A679 (2001) 616

[14] D. Lissauer and E. V. Shuryak, Phys. Lett. B253 (1991) 15

[15] M. Asakawa and C. M. Ko, Phys. Lett. B322 (1994) 33

[16] W. S. Chung, G. Q. Li and C. M. Ko, Nucl. Phys. A625 (1997) 347

[17] C.J. Batty, E. Friedman and A. Gal, Phys. Rep. 287 (1997) 385

[18] S. Hirenzaki, Y. Okumura, H. Toki, E. Oset and A. Ramos, Phys. Rev. C61 (2000) 055205

[19] A. Baca, C. García-Recio and J. Nieves, Nucl. Phys. A673 (2000) 335

[20] A. Gal, nucl-th/0101010.

[21] D.B. Kaplan and A.E. Nelson, Phys. Lett. B175 (1986) 57

[22] E. Anciant et al., hep-ex/0006022

[23] T. Nakano et al., Nucl. Phys. A670 (2000) 332.

[24] A. I. Titov, Y. Oh, S. N. Yang and T. Morii, Phys. Rev. C58 (1998) 2429

[25] S.K. Singh and E. Oset, Phys. Rev. C48 (1993) 1246

[26] T.S. Kosmas and E. Oset, Phys. Rev. C53 (1996) 1409

[27] R.C. Carrasco and E. Oset, Nucl. Phys. A536 (1992) 445

[28] A. Ramos and E. Oset, Nucl. Phys. A671 (2000) 481

[29] J. Schaffner-Bielich, V. Koch and M. Effenberger, Nucl. Phys. A669 (2000) 153

[30] M. Lutz, Phys. Lett. B426 (1998) 12 
[31] R.L. Anderson et al., Phys. Rev. D1 (1970) 27; H.J. Besch et al., Nucl. Phys. B70 (1974) 257; H.J. Berends et al., Phys. Lett. B56 (1975) 408; H.J. Berends et al., Nucl. Phys. B144 (1978) 22; D.P. Barber et al., Z. Phys. C12 (1982) 1; J. Ballam et al. ,Phys. Rev. D7 (1973) 3150

[32] J.M. Laget and R. Mendez-Galain, Nucl. Phys. A581 (1995) 397

[33] V. Koch, Phys. Lett. B337 (1994) 7

[34] T. Waas and W. Weise, Nucl. Phys. A625 (1997) 287

[35] J. Caro, N. Kaiser, S. Wetzel and W. Weise, Nucl. Phys. A672 (2000) 249 